\title{
Off-pump coronary artery bypass graft surgery reduces postoperative AKI risk
}

New research has demonstrated that use of the off-pump coronary artery bypass graft (CABG) technique in cardiac surgery reduces the occurrence of postoperative acute kidney injury (AKI) compared with on-pump CABG surgery. However, no difference in renal function at 1 year was apparent between the two techniques.

Postoperative AKI following cardiac surgery can occur in up to $30 \%$ of patients. To investigate the potential contribution of the cardiac intervention, 2,932 patients were enrolled in a kidney function substudy of the CORONARY trial that compared on-pump and off-pump CABG. In the off-pump group, AKI was detected by increased serum creatinine in $17.5 \%$ of patients versus $20.8 \%$ of patients in the on-pump group $(P=0.01)$. Offpump CABG surgery has shown reduced postoperative AKI risk in various smaller clinical trials, and CORONARY - the largest trial to date in this area-supports these findings.
However, no significant differences in estimated glomerular filtration rate were detected between trial groups (off-pump group $17.1 \%$, on-pump group $15.3 \%$ ) at 1 year, even in patients with pre-existing renal disease. This finding suggests that, although AKI predicts reduced kidney function 1 year later in observational studies, an intervention that reduces mild AKI does not necessarily preserve longer-term kidney function.

The authors concluded that there is no significant difference between off-pump or on-pump CABG surgery in terms of longterm kidney function, and that significant differences in kidney function at $>1$ year are unlikely given the findings at 1 year.

\section{Peter Sidaway}

Original article Garg, A. X., et al. Kidney function after off-pump or on-pump coronary artery bypass graft surgery: a randomized clinical trial. JAMA doi:10.1001/ jama.2014.4952 